\title{
Axl receptor tyrosine kinase is a potential therapeutic target in renal cell carcinoma
}

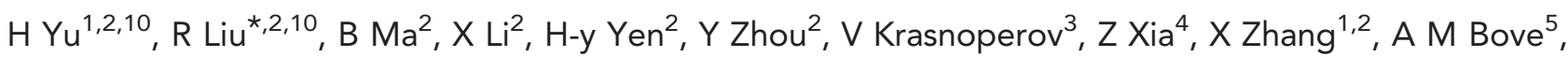
M Buscarini ${ }^{5}$, D Parekh ${ }^{6}$, I S Gill ${ }^{7}$, Q Liao ${ }^{8}$, M Tretiakova $^{9}$, D Quinn ${ }^{2}$, J Zhao ${ }^{1,11}$ and P S Gill ${ }^{\star, 2,11}$

${ }^{1}$ Xiangya Hospital, Central South University, Changsha, Hunan 410008, People's Republic of China; ${ }^{2}$ Department of Medicine, University of Southern California, Norris Hospital, NOR 6332, 1441 Eastlake Avenue, Los Angeles, CA 90033, USA; ${ }^{3}$ Vasgene Therapeutics Inc., Los Angeles, CA 90033, USA; ${ }^{4}$ The State Key Laboratory of Medical Genetics and School of Life Sciences, Central South University, Changsha, Hunan 410078, People's Republic of China; ${ }^{5}$ Department of Urology, Campus Bio-Medico University, Rome 00128, Italy; ${ }^{6}$ Division of Surgery, University of Southern California, Los Angeles, CA 90033, USA; ${ }^{7}$ Department of Urology, University of Southern California, Los Angeles, CA 90033, USA; ${ }^{8}$ Department of Orthopedic Surgery, Xiangya Hospital, Central South University, Changsha, Hunan 41008, People's Republic of China and ${ }^{9}$ Department of Pathology, University of Washington, Seattle, WA 98195, USA

Background: Axl plays multiple roles in tumourigenesis in several cancers. Here we evaluated the expression and biological function of Axl in renal cell carcinoma (RCC).

Methods: Axl expression was analysed in a tissue microarray of 174 RCC samples by immunostaining and a panel of 11 normal tumour pairs of human RCC tissues by western blot, as well as in RCC cell lines by both western blot and quantitative PCR. The effects of Axl knockdown in RCC cells on cell growth and signalling were investigated. The efficacy of a humanised Axl targeting monoclonal antibody hMAb173 was tested in histoculture and tumour xenograft.

Results: We have determined by immunohistochemistry (IHC) that Axl is expressed in $59 \%$ of RCC array samples with moderate to high in 20\% but not expressed in normal kidney tissue. Western blot analysis of 11 pairs of tumour and adjacent normal tissue show high Axl expression in 73\% of the tumours but not normal tissue. Axl is also expressed in RCC cell lines in which Axl knockdown reduces cell viability and PI3K/Akt signalling. The Axl antibody hMAb173 significantly induced RCC cell apoptosis in histoculture and inhibited the growth of RCC tumour in vivo by 78\%. The hMAb173-treated tumours also had significantly reduced Axl protein levels, inhibited PI3K signalling, decreased proliferation, and induced apoptosis.

Conclusions: Axl is highly expressed in RCC and critical for RCC cell survival. Targeting Axl is a potential approach for RCC treatment.

According to the National Cancer Institute statistics, there are $>300000$ people currently living with kidney cancer in the United States. In 2013, 65000 people were diagnosed with kidney cancer and 13600 people died of this disease (Siegel et al, 2013). Renal cell carcinoma (RCC) is the most common type of kidney cancer. About 9 out of 10 kidney cancers are RCCs. Renal cell carcinoma is often treated by surgery and targeted therapy (Chung et al, 2003; Gustafsson et al, 2009a) as it is relatively resistant to standard radiation therapy and cytotoxic chemotherapy (Deschavanne and Fertil, 1996; Ku et al, 2014). Increasing attention has been drawn to biologic therapies and immunodulatory therapies, including small-molecule kinase inhibitors and antibodies (Kanesvaran and

\footnotetext{
*Correspondence: Dr R Liu; E-mail: renliu@usc.edu or Dr PS Gill; E-mail: parkashg@usc.edu

${ }^{10}$ These authors contributed equally to this work.

${ }^{11} \mathrm{~J}$ Zhao and PS Gill are co-senior authors.
}

Received 16 April 2015; revised 4 June 2015; accepted 5 June 2015; published online 16 July 2015 
Tan, 2014). Over the past decade, the US Food and Drug Administration has approved several drugs targeting vascular endothelial growth factor and mammalian target of rapamycin pathways, including sorafenib (Escudier et al, 2007a), sunitinib (Motzer et al, 2007), bevacizumab (Escudier et al, 2007b), temsirolimus (Hudes et al, 2007), everolimus (Motzer et al, 2010), pazopanib (Sternberg et al, 2013), and axitinib (Motzer et al, 2013). These drugs have shown better clinical outcomes than the traditional therapies, but most cancers exhibit drug resistance within a year on drug and the drugs have significant side effects (Harada et al, 2013). Therefore, other effective and safe therapeutics are needed to improve the outcome.

Axl is a receptor tyrosine kinase (RTK) that belongs to TAM family. The other two members of TAM family are Tyro3 and Mer. The TAM kinases have two N-terminal immunoglobulin-like domains and two fibronectin type III repeats in their extracellular regions, a single-pass transmembrane domain, and an intracellular tyrosine kinase domain (Janssen et al, 1991; O'Bryan et al, 1991; Lai et al, 1994; Lemke, 2013). The ligand for TAM kinases is vitamin K-dependent growth arrest-specific gene 6 (Gas6). Gas6 binding to Axl induces Axl dimerisation and autophosphorylation, leading to signal transduction (Lemke, 2013).

Axl is overexpressed in many human cancers (Ye et al, 2010) including breast (Meric et al, 2002), lung (Shieh et al, 2005), colon (Bosurgi et al, 2013), prostate (Paccez et al, 2013), gastric (Wu et al, 2002), pancreatic (Leconet et al, 2013), Kaposi's sarcoma (Liu et al, 2010), acute myeloid leukaemia (Ben-Batalla et al, 2013), as well as renal cancer (Chung et al, 2003; Gustafsson et al, 2009b). Axl oncogenic signalling promotes cancer cell survival, proliferation, migration, and invasion (Han et al, 2013). It is recently reported that activation of Axl is required for erlotinib resistance in epidermal growth factor receptor (EGFR)-mutant non-small-cell lung carcinoma (NSCLC) tumour (Zhang et al, 2012), and thus targeting Axl may prevent or overcome acquired resistance to EGFR tyrosine kinase inhibitors (TKIs) in individuals with EGFR-mutant lung cancer. The signals downstream of Axl are mitogen-activated protein kinases (MAPKs)/extracellular signal regulated kinases (ERKs) or phosphatidylinositol 3 kinase (PI3K)/Akt pathway depending on cancer type (Melaragno et al, 1999; Collett et al, 2007). Recent work in genetic models suggests that TAM kinases play an key role in innate immunity (Zhang et al, 2013), and the Janus kinase/ signal transducer and activator of transcription (STAT) pathway mediates this function (Rothlin et al, 2007).

Induction of Axl mRNA in RCC has been reported previously (Chung et al, 2003; Gustafsson et al, 2009a). However, Axl protein expression status and its role in RCC remain unknown. In the current study we show that Axl protein is overexpressed in human RCC tissues and established RCC cell lines, and it is a survival factor for RCC cells. Targeting Axl with our novel monoclonal antibody hMAb173 induced RCC cell apoptosis ex vivo and in vivo. These results suggest that $\mathrm{Axl}$ is a potential therapeutic target in RCC.

\section{MATERIALS AND METHODS}

Antibodies and other reagents. Antibodies against human Axl, Mer, cleaved caspase 3, Akt, phosphorylated Akt (Ser473), STAT3, phosphorylated STAT3 (Tyr705), Erk1/2, phosphorylated Erk1/2 (Thr202/Tyr204), ribosomal protein S6, and phosphorylated S6 (Ser235/236) were from Cell Signaling (Danvers, MA, USA). The Tyro3 and Gas6 antibodies were from R\&D Systems (Minneapolis, MN, USA). $\beta$-Actin antibody was from Sigma-Aldrich (St Louis, MO, USA). Ki67 antibody was from Abcam (Cambridge, MA, USA). IRdye800/700 and horseradish peroxidase (HRP)-conjugated secondary antibodies were from Rockland Immunochemicals (Gilbertsville, PA, USA). The TdT-mediated dUTP nick-end labelling (TUNEL) assay kit was from Promega (Madison, MI, USA). Protease inhibitor cocktail was from Thermo Scientific (Waltham, MA, USA).

Cell culture. The human kidney cancer cell lines 786-O, 769-P, ACHN, human embryonic kidney cell line 293T, human colorectal cancer cell HT29, human lung cancer cell line A549, and human breast cancer cell line MDA-MB-231 were obtained from American Type Culture Collection (ATCC, Manassas, VA, USA). All cell lines were cultured following the instructions of ATCC.

RCC samples in tissue microarray (TMA) cohort. Our study group consisted of 174 nephrectomy specimens, including 110 clear cell RCCs (CCRCCs) and 64 papillary RCCs (PRCCs), as well as 4 normal kidney tissues. The H\&E slides from each case were reviewed by experienced pathologist to select representative sections for TMA fabrication with approval from the University of Washington Institutional Review Board and signed informed consent. From each case a minimum of three tissue cylinders with a diameter of 1 or $1.5 \mathrm{~mm}$ were arrayed into a recipient block using either manual or automated tissue microarrayer ATA-27 (Beecher Instruments, Sun Prairie, WI, USA). The $4-\mu \mathrm{m}$ thick serial sections were subjected to immunohistochemistry (IHC) analysis as outlined below.

Frozen RCC samples. A total of 11 pairs of primary tumour and adjacent normal tissues were surgically attained from RCC patients and kept frozen. The pathological status of tissues was confirmed with H\&E staining and pathological examination. These RCC patients include 10 cases of clear cell carcinoma and one case of chromophobic carcinoma. All cases were in stage II or III. The ethical use of the human tissues for research was approved by the University of Southern California Institutional Review Board and tissues were collected after signed informed consent was obtained.

Immunohistochemistry. Paraffin RCC tissue sections were deparaffinised in xylene, rehydrated through graded ethanol solutions, and washed in distilled water. Antigen was retrieved by heating sections in citrate buffer ( $\mathrm{pH} \mathrm{6)}$ for $20 \mathrm{~min}$ at $95^{\circ} \mathrm{C}$, followed by cooling for $20 \mathrm{~min}$ at room temperature. Endogenous peroxidase activity was quenched by incubation in $3 \% \mathrm{H}_{2} \mathrm{O}_{2}$ in $\mathrm{H}_{2} \mathrm{O}$ for 5 min. The sections were then incubated with anti-Axl antibody (rabbit monoclonal, Cell Signaling clone C89E7; $1: 1000$ dilution) at $4{ }^{\circ} \mathrm{C}$ for overnight, with anti-rabbit amplifier antibody (Vector Laboratories, Burlingame, CA, USA) at room temperature for $30 \mathrm{~min}$, and finally with ImmPress Excel polymer reagent (Vector Laboratories) at room temperature for $30 \mathrm{~min}$. Signal was developed using ImmPACT DAB EqV working solution (Vector Laboratories) and pictures were taken with an Olympus BX51 microscope (Olympus USA, Center Valley, PA, USA) equipped with a Retiga 200R camera (Qimaging, Surrey, BC, Canada).

Cytoplasmic and membranous staining were scored in a semiquantitative manner where positivity of $>5 \%$ of the tumour cells was necessary for scoring a case as positive. Staining intensity in positive cases was graded on a scale of $1-3$, where a staining intensity of 2 or 3 was considered high. The mean value was recorded for each individual case as the average intensity of all three TMA cores.

Western blot. Frozen RCC samples were sectioned at $20 \mu \mathrm{m}$ and lysed on ice with RIPA buffer (Thermo Scientific) for $20 \mathrm{~min}$. Lysate was centrifuged at 13000 r.p.m. for $15 \mathrm{~min}$ at $4{ }^{\circ} \mathrm{C}$ and the supernatant was transferred to a fresh tube. Protein concentration was qualified by BCA Protein Assay (Thermo Scientific). Approximately $10 \mu \mathrm{g}$ of whole-tissue lysate was run on $4-20 \%$ Tris-glycine gradient gel (Bio-Rad, Hercules, CA, USA) and transferred onto nitrocellulose membrane (Bio-Rad). The membrane was blocked with $5 \%$ nonfat dry milk in Tris-buffered saline for $1 \mathrm{~h}$, and then incubated with $1 \mu \mathrm{g} \mathrm{ml}^{-1}$ primary antibody at 
$4{ }^{\circ} \mathrm{C}$ overnight. Membrane was then incubated with fluorescent secondary antibodies for $1 \mathrm{~h}$ and signal was detected using Odyssey System (LI-COR, Lincoln, NE, USA).

For western blot analysis of RCC cells, cells were grown on six-well plates (BD Biosciences, San Jose, CA, USA) and lysed on ice with Laemmli Sample buffer (Bio-Rad). Equal amount of lysates were separated by SDS-PAGE and then processed as described above.

Quantitative PCR (qPCR). RNA was extracted from cell lines using QIAGEN RNAeasy RNA isolation kit (Qiagen, Valencia, CA, USA) and reverse transcribed into cDNA using Thermo Scientific cDNA synthesis kit. The primers for amplification were: Axl-F, 5'-TGGAGGAGCCCGAAGACAGGACT-3'; Axl-R, 5'-GAGAG GCCGGGGTGAATGGAG-3'; Mer-F， 5'-GATTGGAGACAGGA CCAAAGC-3'; Mer-R, 5'-GACGTAAATAACGTCTGCTTGG- ${ }^{\prime}$; Tyro3-F, 5'-CCATATGCTGGCATCGAAAAC-3'; Tyro3-R, 5'-A TCCCTGCCAGGTAGCTCCA-3'; Gas6-F， 5'-AGAACCTGCCC GGCTCCTACTCCT-3'; Gas6-R, 5'-AGCCCCACTGTTCCCATC ACTG- $3^{\prime} ; \beta$-actin-F, $5^{\prime}$-TCTGAAGTCGGCAAGGGGGAGTGA$3^{\prime}$; and $\beta$-actin-R, $5^{\prime}$-CGTCGCGCCGCTGGGTTTTAT- $3^{\prime}$. Quantitative PCR was performed with Bio-Rad SYBR Green mastermix on LightCycler 480 (Roche, Indianapolis, IN, USA). Each amplification reaction was checked for the absence of nonspecific PCR products by melting curve analysis. The threshold cycle numbers obtained from qPCR were compared to generate the relative copy number as described by Livak and Schmittgen (2001). Data were normalised against $\beta$-actin, and then relative gene expression in cancer cells was compared with MDA-MB-231 cell.

Axl siRNA and transfection. Prevalidated Axl siRNA and nontargeting control siRNA were purchased from QIAGEN. The sense strand sequence of Axl siRNA is $5^{\prime}$-CAAGAUUCUAGAUGAUUAATT- $3^{\prime}$. The sequence of the control siRNA is not disclosed by the vendor. We used Lipofectamine RNAiMAX (Invitrogen, Carlsbad, CA, USA) for siRNA transfection following the manufacturer's protocol. At $72 \mathrm{~h}$ after transfection, we collected whole-cell lysates for western blot or analysed viable cell number using 3-(4,5-dimethylthiazol-2-yl)-2,5-diphenyltetrazolium bromide (MTT) assay.

Cell viability assay. Cells were seeded in 24-well plates at a density of $2 \times 10^{4}$ cells per well in $500 \mu \mathrm{l}$. The medium was changed to $2 \%$ FBS medium after cells were attached, followed by siRNA transfection in triplicate. At 3 days after transfection, cell viability was assessed by MTT assay as described previously (Liu et al, 2010).

Cell invasion assay. Cell invasion assay was carried out as previously described (Liu et al, 2010). Briefly, after transfection with control siRNA or Axl siRNA (20 nM) for $24 \mathrm{~h}, 5 \times 10^{4}$ cells were collected and seeded in $500 \mu \mathrm{l}$ serum-free medium on the upper well of the transwell plate(BD Bioscience) and migration was induced by adding $750 \mu \mathrm{l}$ medium with $10 \%$ FBS in the bottom well. After overnight incubation at $37^{\circ} \mathrm{C}$, the cells on the outer surface of the membrane were fixed and stained with Diff Quick (Dade Behring, Newark, DE, USA) and counted with an Olympus BX51 microscope (Olympus USA) equipped with a Retiga 200R camera (Qimaging). Experiments were performed in duplicate and at least three pictures were taken from each membrane.

Antibody endocytosis. Cells cultured on 4-well chamber slide (BD Bioscience) were incubated with $10 \mu \mathrm{g} \mathrm{ml}{ }^{-1} \mathrm{hMAb} 173$ at $4{ }^{\circ} \mathrm{C}$ or $37^{\circ} \mathrm{C}$ for $1 \mathrm{~h}$, fixed with $4 \%$ paraformaldehyde for $20 \mathrm{~min}$ at $4{ }^{\circ} \mathrm{C}$, and washed with cold PBS for 3 times. Cells were then permeabilised with $0.1 \%$ Triton X-100, washed with PBS for 3 times, and stained with Alexa Fluor 488-conjugate anti-human IgG antibody (Invitrogen) for $30 \mathrm{~min}$ at room temperature. Nuclei were counterstained with 6-diamidino-2-phenylindole dihydrochloridehydrate (DAPI). Images were obtained with a confocal microscope (Zeiss, Thornwood, NY, USA).
Immunofluorescence. Fresh frozen tissue embedded in OCT was sectioned at $5 \mu \mathrm{m}$ and fixed in phosphate-buffered $4 \%$ paraformaldehyde and washed in PBS. Sections were then incubated with primary antibodies ( $1: 1000$ dilution) overnight at $4{ }^{\circ} \mathrm{C}$. After washing with PBS, antibody binding was localised with appropriate Alexa Fluor-conjugated secondary antibodies (Invitrogen; 1:250 dilution). Nuclei were counterstained with DAPI. Images were obtained with a Nikon Eclipse 80i fluorescence microscope (Melville, NY, USA) equipped with a CoolSnap ES camera (Photometrics, Tucson, AZ, USA).

Histoculture. Freshly harvested kidney tumour was dissected to $\sim 2 \mathrm{~mm}$ thick slices and placed on tissue culture insert (Millipore, Billerica, MA, USA). Culture medium (RPMI supplemented with $10 \%$ FBS) was added to just reach the tissue slices and exposed to air on the top. Tissues were treated with $10 \mu \mathrm{g} \mathrm{ml}^{-1}$ control human IgG (Rockland, Gilbertsville, PA, USA) or hMAb173 for 2 days, followed by OCT embedding and immunostaining. At least two tissue samples were treated in each group.

Murine tumour xenograft models. The 786-O and HT29 cells were propagated, collected after trypsin digestion, and resuspended in serum-free medium. The $5 \times 10^{6} 786-\mathrm{O}$ cells or $2 \times 10^{6}$ HT2 9 cells were injected subcutaneously in the flanks of male 8-week-old $\mathrm{Balb} / \mathrm{C}$ nu/nu mice. Tumour growth was measured 3 times a week, and volume was estimated as $0.52 \times$ length $\times$ width $^{2}$. Once tumours were established $\left(\sim 100 \mathrm{~mm}^{3}\right)$, animals were distributed into hMAb173 and control IgG (Rockland) groups $(n=8)$. Each group was treated by intraperitoneal injection of $20 \mathrm{mg} \mathrm{kg}^{-1}$ of antibody 2 times a week. At the end of the experiment, mice were killed for tissue analysis. All procedures were approved by University of Southern California institutional animal care and use committee and performed in accordance with the Animal Welfare Act regulations.

Statistics. For viability assay and invasion assay, replicate data points were averaged and then compared between groups using unpaired, two-tailed Student's $t$-test. For 786-O tumour xenograft study, only the tumour volumes at the last time point were compared between groups using unpaired, two-tailed Student's $t$ test. All experiments above were repeated at least twice and similar results were obtained. For analysis of immunofluorescent staining, at least three pictures were taken and percentages of positive cell of each picture were averaged and compared between groups using unpaired, two-tailed Student's $t$-test. All error bars in the experiments performed in this study represent s.d. unless specified in the figure legend.

\section{RESULTS}

Overexpression of Axl in RCC tissues. To study Axl expression in RCC, we analysed an array of 174 RCC samples by IHC staining. Axl expression was detected in 54 of 110 cases (49\%) of clear cell RCCs with moderate and strong staining intensity in $26 \%$ of positive cases (Figure 1A). Positive tumours exhibited fine granular cytoplasmic staining pattern with membranous accentuation (Figure 1B). Papillary RCCs were positive in 49 of 64 (77\%) with moderate to strong staining in $41 \%$ of all positive cases (Figure 1A). Axl expression in PRCC was also cytoplasmic and finely granular (Figure 1B). Overall, there are $20 \%$ of RCC cases with moderate to high Axl expression. In the majority of clear cell and papillary RCCs, mixed inflammatory infiltrate was present including tumour-infiltrating lymphocytes and foamy macrophages that were also strongly positive for Axl (Supplementary Figure S1). Scoring of tumour cells did not include inflammatory component that was substantial in $40 \%$ of cases. Normal kidney tissues were negative in all 4 cases. 
A

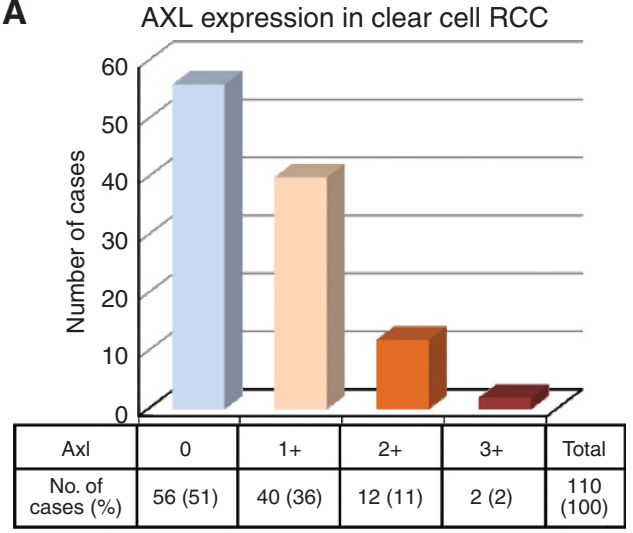

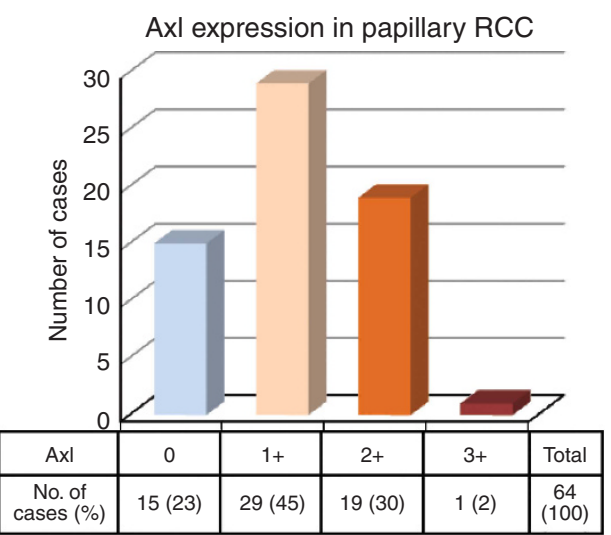

B
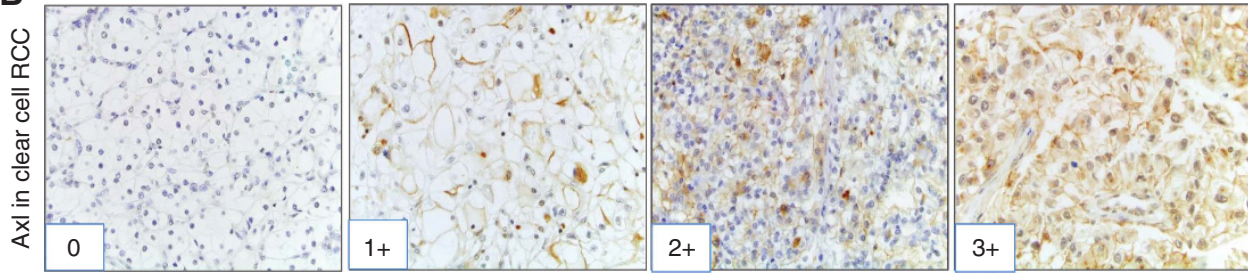
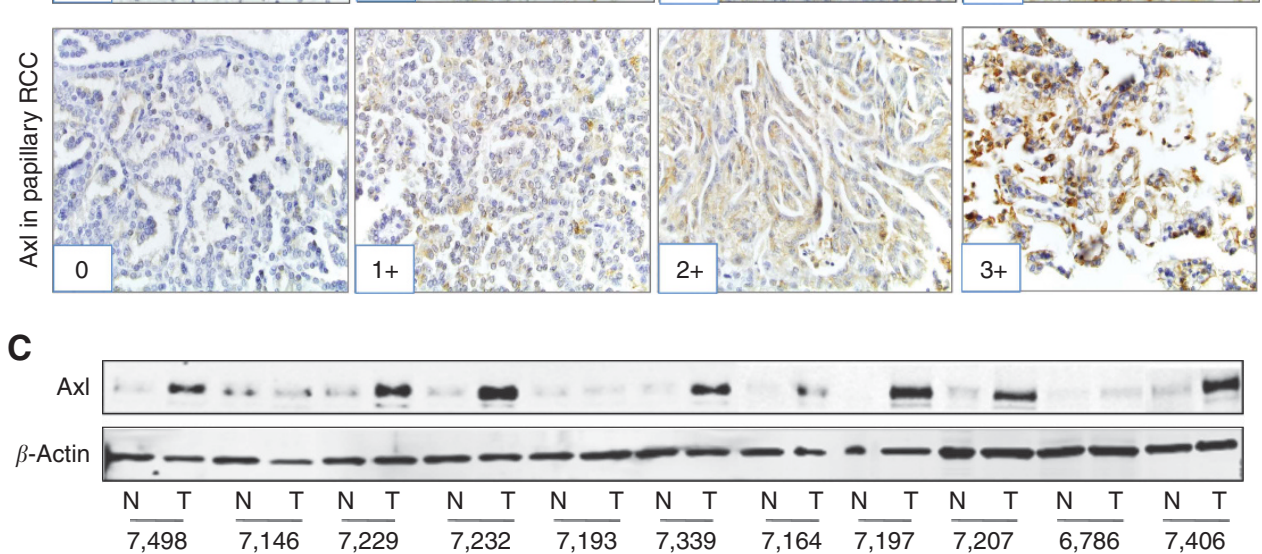

Figure 1. Overexpression of $\mathrm{Axl}$ in renal cancer. (A) Axl expression was analysed in an array of $174 \mathrm{RCC}$ samples with immunohistochemistry staining. The number of cases with no $(0)$, weak $(1+)$, moderate $(2+)$, or strong $(3+)$ Axl expression is shown. Left, clear cell RCC; right, papillary RCC. (B) Representative pictures of IHC analysis of Axl expression in clear cell RCC (top) and papillary RCC (bottom) tissue array. Axl is in brown. (C) Kidney tumour (T) and adjacent normal kidney tissue $(\mathrm{N})$ were collected in pairs. Samples were lysed and used for western blot to evaluate Axl expression. $\beta$-Actin was probed to ensure equal loading.

We also examined Axl expression in 11 pairs of frozen normal and tumour tissue samples by western blot (each pair derived from the same RCC patient). Out of 11 samples, 8 (73\%) showed high Axl expression in tumour and low/no Axl expression in the adjacent normal tissue (Figure 1C). There was low/no Axl expression in both normal and tumour tissues in the remaining three pairs of samples. We also examined the expression of the other two TAM receptors Mer and Tyro3 in six pairs of tissues that had AXL overexpression in tumour. Mer or Tyro3 induction in tumour was only found in one pair and they were not coexpressed (Supplementary Figure S2).

The specificity of the Axl antibody used for IHC and western blot was examined by immunocytometry staining of cancer cells with or without Axl. As shown in Supplementary Figure S3A, Axl antibody stained Axl-positive 786-O cells (Figure 2) treated with control siRNA, but failed to stain 786-O cells treated with Axl siRNA as well as Axl-negative HT29 cells (Figure 2B). In addition, the complete western blot for Figure 1C is included as Supplementary Figure S3B, demonstrating high specificity of the Axl antibody used in this study.
Overexpression of Axl in RCC cell lines. In light of the overexpression of Axl in human RCC tissues, we checked Axl expression in three RCC cell lines: 786-O, 769-P, and ACHN. We used human breast cancer cell MDA-MB-231 known to have high Axl expression (Zhang et al, 2008; Gjerdrum et al, 2010) and embryonic kidney cell 293T as positive and negative controls, respectively. Quantitative RT-PCR showed high Axl expression in all three RCC cell lines, with levels similar to MDA-MB-231 and more than 1000-fold over 293T (Figure 2A). We also analysed the expression of the other two TAM receptors Mer and Tyro3 and their cognate ligand Gas6. In sharp contrast to Axl, the expression of Mer and Tyro3 in cancer cells was much lower than in 293T cells, and similar level of Gas6 expression was found in both the cancer and normal cells (Figure 2A). This is consistent with the western blot results, in which Axl was the only TAM kinase highly induced in all RCC cell lines compared with 293T (Figure 2B). Mer was hardly expressed in any RCC cell lines, whereas Tyro3 only had modest expression in 769-P and low/no expression in the other two cell lines. Meanwhile, Gas6 protein expression was absent in only 786-O (Figure 2B). 
A

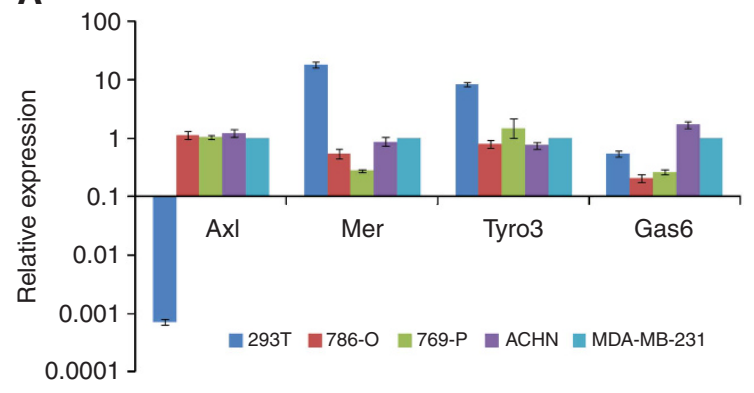

B

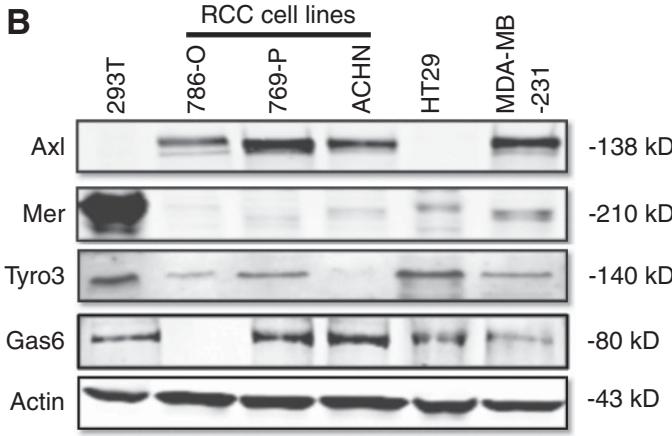

Figure 2. Overexpression of Axl in RCC cell lines. (A) The expressions of TAM kinases and Gas6 in RCC cell lines 786-O, 769-P, and ACHN were analysed with quantitative RT-PCR. Gene expression was first normalised against $\beta$-actin and then compared with breast cancer cell line MDA-MB231. Normal human embryonic kidney cell line 293T was included as control. Axl is the only TAM kinase highly induced in RCC cell. Error bars represent s.d. (B) The protein expression profile of TAM kinases and Gas6 in RCC cells was analysed with western blot. An Axl-negative colorectal cancer cell HT29 was used as a control. Whole-cell lysate was analysed and $\beta$-actin was probed to show equal loading. Protein size is shown on the right of each panel. These experiments were repeated at least twice and similar results were obtained.

Knockdown of Axl in RCC cells induced cell death, inhibited invasion, and decreased PI3K signalling. To understand the function of Axl in RCC, we examined the effect of Axl knockdown in RCC cells in vitro. We transfected 786-O, ACHN, and 293T cells with Axl siRNA and analysed cell signalling change and viable cell number 3 days after transfection. In both 786-O and ACHN, Axl siRNA effectively decreased Axl expression even at $20 \mathrm{~nm}$ concentration (Figure 3A). We also noted that many cancer cells were nonviable and detached from the plates after Axl knockdown. The viable cell analysis (MTT assay) indicated that Axl knockdown significantly reduced the viable cell number of two RCC cells but not 293 T cells (Figure 3B).

We next studied the effect of Axl knockdown on RCC cell invasion in a transwell invasion assay. As shown in Figure 3C, compared with control siRNA-treated cells, Axl siRNA-treated cells had significantly fewer cells invading through matrigel $(P<0.002)$.

These results suggest that Axl promotes RCC cell survival/ invasion that could be through PI3K or MAPK pathway. We thus analysed the signalling pathways affected by Axl knockdown using western blot. We found that the levels of phosphorylated Akt and S6, the two key signal molecules of PI3K pathway, were markedly reduced after Axl knockdown in 786-O cell (Figure 3D and E), whereas both phosphorylated STAT3 and ERK1/2 did not change (Figure 3D and E). Similar results were obtained with the other two RCC cell lines (data not shown). Therefore, PI3K is likely the major signalling pathway downstream to Axl in renal cancer cells.

An Axl degrading antibody hMAb173 induced kidney cancer cell apoptosis ex vivo. We have developed a monoclonal antibody against Axl that specifically binds to Axl extracellular domain and induces Axl endocytosis and degradation, and hence inhibits Axlmediated cell growth and invasion previously shown in Kaposi's sarcoma cells (Liu et al, 2010). In order to safely administer MAb173 in humans we have humanised MAb173 to prevent immunogenicity. We analysed humanised clones in silico to avoid potential T-cell epitopes in variable $\mathrm{V}$ regions. Lack of immunogenicity in the lead humanised antibody for antigen was confirmed by measuring $\mathrm{T}$-cell responses against the whole antibody compared with the murine antibody ex vivo. The results demonstrated that the fully humanised antibody hMAb173 has no reactivity compared with the parent murine monoclonal antibody that has high (60\% donor response rate) reactivity (Supplementary Figure S4). Thus, hMAb173 has a very low likelihood of triggering an immunogenic response. This antibody also retains affinity and efficacy comparable to the parental murine antibody, and has half-life more than 3 days in mouse (data not shown). In a non-GLP toxicology study in mice, hMAb173 did not show any toxicity to major organs except inflammation in pancreas that was also observed in control antibody-treated animals (data not shown). However, as hMAb173 does not recognise mouse Axl, its toxicity profile needs to be further evaluated in monkeys whose Axl protein binds hMAb173.

As Axl is a survival factor in RCC cells, we tested hMAb173 as a potential Axl targeting therapeutic in RCC. We first checked the activity of hMAb173 in inducing Axl endocytosis in RCC cells. At $4{ }^{\circ} \mathrm{C}$, hMAb173 localised to the surface of 786-O (Figure 4A, left). However, the antibody was endocytosed when incubated with 786$\mathrm{O}$ for $1 \mathrm{~h}$ at $37^{\circ} \mathrm{C}$ (Figure $4 \mathrm{~A}$, right). Consistently, hMAb173 efficiently degraded Axl as shown by western blot (Figure 4B). It reduced Axl protein expression by $80 \%$ at $3 \mathrm{~h}$ and $90 \%$ at $48 \mathrm{~h}$.

We then tested hMAb173 in an ex vivo tumour model. Freshly harvested human RCC tumour was dissected into small pieces and grown in inserts submerged in culture medium. Control antibody and hMAb173 were added into culture medium and tumours were analysed 2 days later. As shown in Figure 4C, hMAb173 significantly induced apoptosis (manifested by cleaved caspase 3 staining), whereas control antibody had no effect.

Inhibition of 786-O xenograft tumour growth by hMAb173. We next evaluated the activity of hMAb173 in vivo in a tumour xenograft model. 786-O was implanted subcutaeously in nude mice and the established tumours were randomly grouped and treated with control IgG or hMAb173 at $20 \mathrm{mg} \mathrm{kg}^{-1}$ twice a week for 4 weeks. This dosing schedule was chosen based on pharmacokinetics studies in mice to maintain sufficient drug level in blood. As shown in Figure 5A, hMAb173 significantly inhibited tumour growth. On day 26 , it inhibited tumour growth by $78 \%(P<0.002)$ compared with control group (Figure 5A). To confirm the antitumour activity of hMAb173 is based on Axl targeting, we tested hMAb173 in an Axl-negative cancer cell model HT29. As shown in Figure 5B, hMAb173 had no effect on the growth of HT29 tumour, indicating that hMAb173 specifically targets Axl and inhibits tumour growth. In both tumour models, no toxicity was observed following hMAb173 therapy.

At the end of the 786-O xenograft experiment, tumours were harvested and analysed by immunostaining. As expected, Axl, detected by an antibody recognising a different epitope (located in the intracelluar domain), was barely detectable in hMAb173treated tumours, whereas it was highly expressed in control IgGtreated tumours (Figure 5B). At the same time, hMAb173 promoted tumour cell apoptosis in vivo leading to $72 \%$ apoptosis in tumour cells, whereas the control antibody-treated tumours had almost no apoptosis (Figure 5C). In addition, 30\% tumour cells of control group showed Ki67 signal (proliferation marker), but 
A
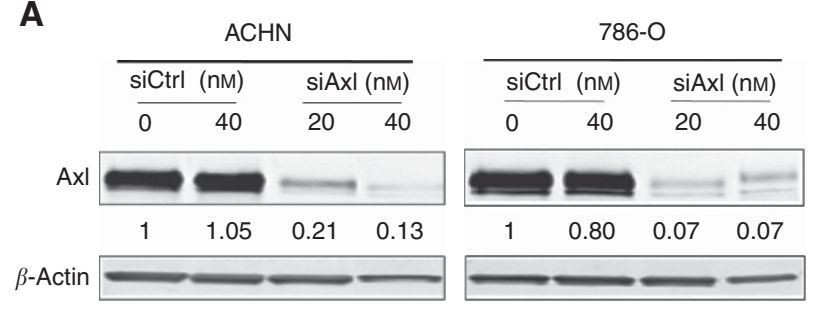

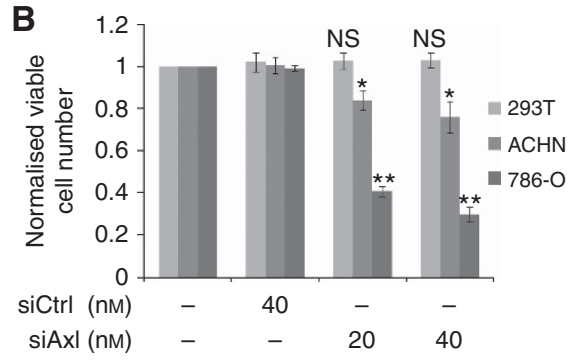

$\mathbf{E}$

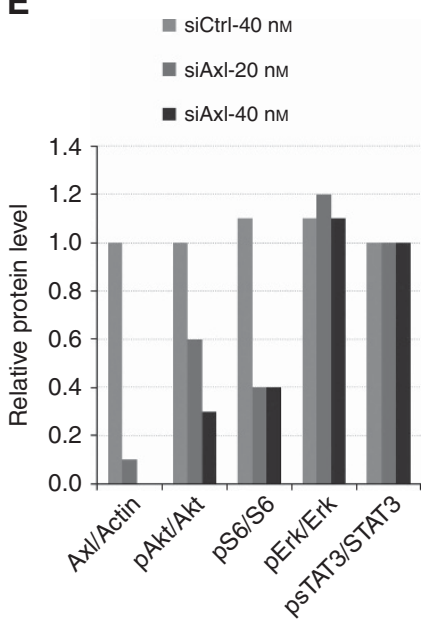

Figure 3. Axl knockdown reduces RCC cell viability and inhibits invasion. (A) Western blot showing high efficacy of Axl siRNA in Axl knockdown in 786-O and ACHN cells. Signal intensity was quantified with ImageJ (NIH, Bethesda, MD, USA) and normalised to $\beta$-actin. (B) Viable cell number after Axl knockdown was analysed with MTT assay. Axl knockdown significantly reduced viability of RCC cells $786-\mathrm{O}$ and $\mathrm{ACHN}$, but not normal cell 293T. Experiment was performed in triplicate. The viability of Axl siRNA-treated cells was compared with $40 \mathrm{~nm}$ control siRNA-treated cells. (C) Representative pictures showing Axl knockdown significantly inhibited invasion of 786-O through matrigel. Experiment was performed in duplicate. At least three pictures from each matrigel insert were taken and invading cells were counted with ImageJ (NIH). In (B) and (C), $P$-value was calculated using two-tailed, unpaired Student's t-test. NS, not significant; ${ }^{*} P<0.05$; ${ }^{\star \star} P<0.002$. (D) Western blot showing Axl knockdown significantly inhibited PI3K signalling (pAkt and pS6 levels), but not MAPK (pERK1/2) and STAT3 (pSTAT3) signallings. (E) Signal intensity in (D) was quantified with ImageJ (NIH). Axl level was normalised to $\beta$-actin and phosphorylated signalling protein levels were normalised to their corresponding total protein levels respectively. All these experiments were repeated at least twice and similar results were obtained. All error bars represent s.d.

almost no signal was found in hMAb173-treated group (Figure 5D). We also investigated the effect of hMAb173 on PI3K signalling. As shown in Figure 5E, pS6 was significantly decreased in hMAb173-treated tumours compared with control group, consistent with our findings in vitro.

\section{DISCUSSION}

Axl is expressed in many cancers (Hafizi and Dahlbäck, 2006). Here we used two different methods to show that Axl is overexpressed in RCC. The IHC analysis of 174 RCC cases showed positive Axl expression in 59\% of the cases with moderate to strong pattern in $20 \%$ of the cases. Papillary RCC has higher Axl positivity compared with clear cell RCC (77\% vs $49 \%$ in overall positivity and $32 \%$ vs $13 \%$ in moderate/high positivity). No Axl expression was found in all four normal kidney tissues included in the study. Consistently, western blot analysis of 11 pairs of tumour and normal kidney tissue also showed high Axl expression in $73 \%$ of the tumours.

Axl expression in RCC has been reported previously. A large study of over 200 patients showed elevated Axl mRNA levels in clear cell carcinoma, and high levels predicted shorter survival (Gustafsson et al, 2009a). However, no correlation between Axl protein in tumour tissue and RCC stage or patient survival was found, even though higher soluble Axl in patient serum correlated with more advanced RCC stage. In another study, Axl mRNA expression was induced in tumour in 20 pairs of tumour and normal kidney tissue (Chung et al, 2003). However, no significant difference in Axl protein expression was found between normal and tumour tissue in a subset of these samples. The discrepancy between the mRNA and protein expression could be explained by the limitation of the sensitivity/specificity of the antibody used.

The significant induction of Axl in renal cancer cells (over 1000fold in mRNA compared with normal kidney cell) is distinct from the two other members of this receptor tyrosine kinase family, namely Mer and Tyro3. In fact, the expression of Mer and Tyro3 in renal cancer cell lines was even lower than the normal cell. Furthermore, Mer and Tyro3 only had protein expression in one of six RCC tumours analysed. These results suggest that Axl is the predominant TAM kinase in RCC. However, to confirm these findings, we need to analyse the expression and function of Mer and Tyro3 in a large set of RCC samples. The TAM kinases have a common ligand Gas6. It was reported that high Gas6 mRNA expression in RCC was associated with less advanced disease stage 

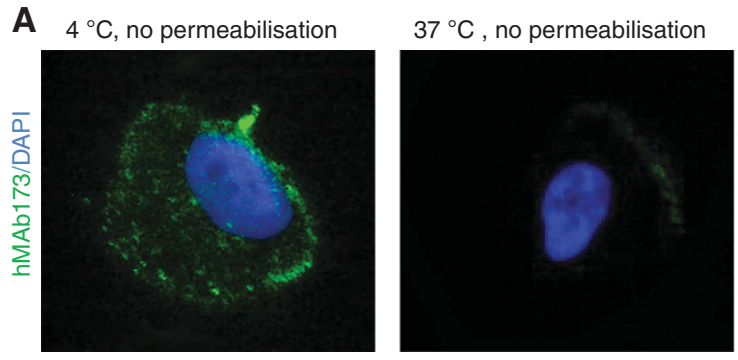

$37^{\circ} \mathrm{C}$, permeabilisation
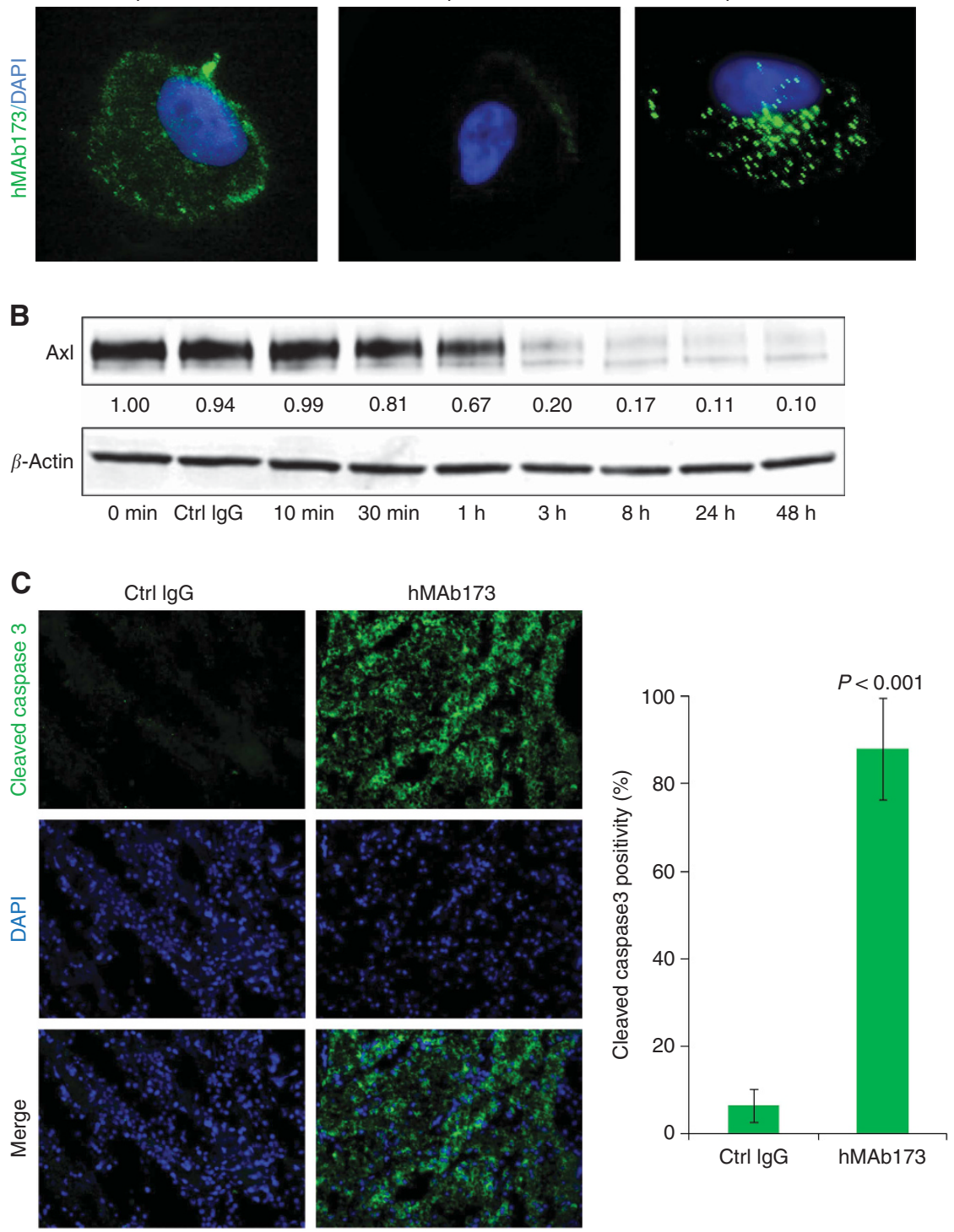

Figure 4. The Axl antibody hMAb173 promotes Axl endocytosis and degradation in vitro and RCC cell apoptosis ex vivo. (A) 786-O cells were incubated with $10 \mu \mathrm{g} \mathrm{ml} l^{-1} \mathrm{hMAb} 173$ at $4{ }^{\circ} \mathrm{C}$ or $37^{\circ} \mathrm{C}$ for $1 \mathrm{~h}$. The cells were fixed with $4 \%$ paraformaldehyde and hMAb173 was localised with fluorochrome-conjugated secondary antibody. Triton X-100 (0.1\%) was used for permeabilisation. Nuclei were counterstained with DAPI. hMAb173 was on $786-\mathrm{O}$ cell surface at $4^{\circ} \mathrm{C}$ but it underwent endocytosis at $37^{\circ} \mathrm{C}$. (B) $786-\mathrm{O}$ cells were treated with $10 \mu \mathrm{g} \mathrm{ml}{ }^{-1} \mathrm{hMAb} 173$ and harvested at a series of time points. Cells treated by control lgG (normal human lgG) were harvested only at the $48 \mathrm{~h}$ time point. Signal intensity was quantified with ImageJ $(\mathrm{NIH})$ and normalised to $\beta$-actin. Experiments in $(\mathbf{A})$ and $(\mathbf{B})$ were repeated at least twice and similar results were observed. (C) Freshly collected human kidney tumours were cultured ex vivo and treated with control IgG and hMAb173 for 2 days. Tumour tissues were processed for immunostaining with antibody specific for cleaved caspase 3. Two tumour tissues from each group and at least three pictures from each tissue were taken for signal quantification using ImageJ. P-value was calculated with two-tailed, unpaired Student's $t$-test.

and improved patient survival (Gustafsson et al, 2009a). However, in the same study, high Gas6 protein level in the serum correlated with more advanced tumour stage (Gustafsson et al, 2009a), leaving the role of Gas6 in RCC still unclear. In the current study, we found Gas6 protein was expressed in normal kidney cell, suggesting Gas6 may not have tumour cell-specific expression. $786-\mathrm{O}$ is a cell line whose survival is highly dependent on Axl but it does not express Gas6, and therefore the cell death induced by Axl knockdown in 786-O in vitro is unlikely related to Gas6-Axl but autocrine signalling. Axl can be activated by mechanisms other than Gas6 such as reactive oxygen species and ligand-independent dimerisation (Hafizi et al, 2002; Konishi et al, 2004). We will further study by which of these mechanisms Axl activation is induced in RCC cells and also the exact role of Gas6 in renal cancer in the future.
Knockdown of Axl in RCC cell lines led to loss of cell viability, indicating that $\mathrm{Axl}$ is a survival factor in RCC. Axl is also important for RCC cell migration. The PI3K/Akt/S6 signalling seems to be the major pathway downstream of Axl, consistent with many previous studies (Goruppi et al, 1999; Liu et al, 2010; Paccez et al, 2013). Others have also reported induction of MAPK or STAT3 downstream of Axl (Fridell et al, 1996; Ou et al, 2011; Yanagita et al, 2001). However, both MAPK and STAT3 did not change significantly after Axl knockdown in the current study, possibly because Axl utilises different signalling pathways in different cancer cells. As discussed above, Axl overexpression has higher percentage in PRCC than CCRCC. One limitation of this study is that all three RCC cell lines tested were CCRCCs. More study on PRCC cell lines will give us better understanding of the role of Axl in RCC. 

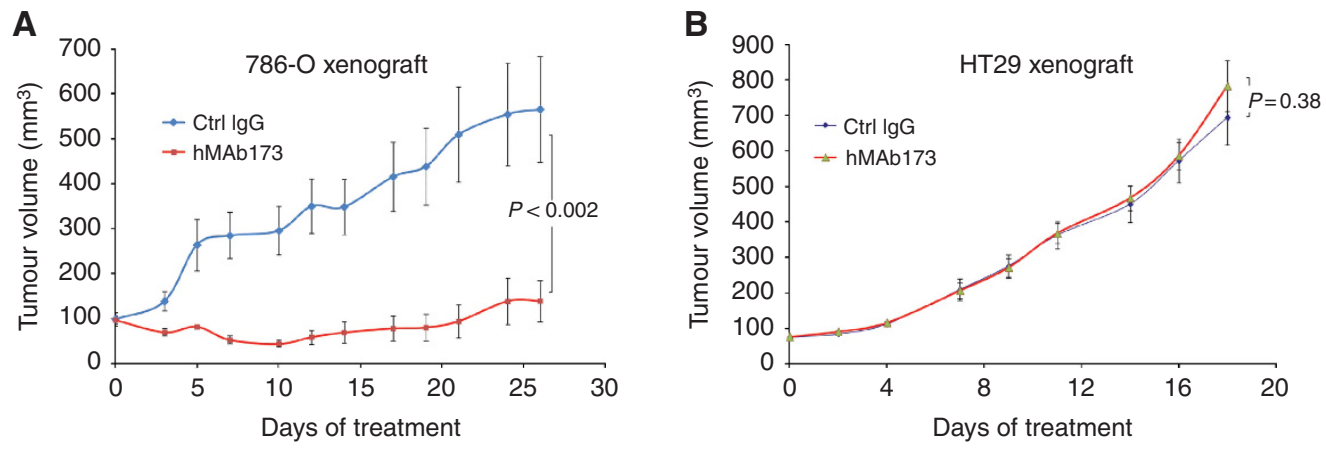

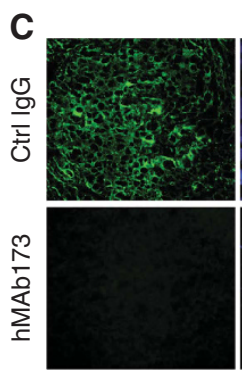

Axl

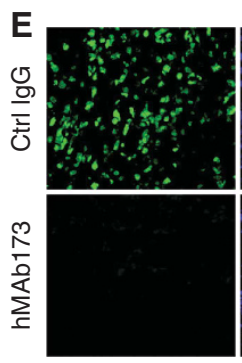

Ki67

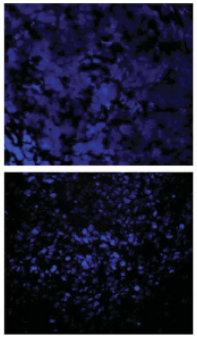

DAPI

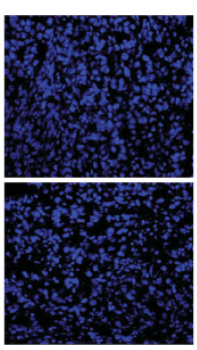

DAPI

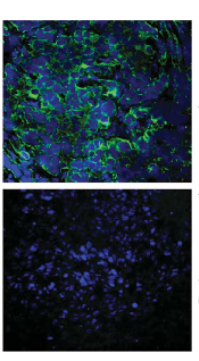

Merge

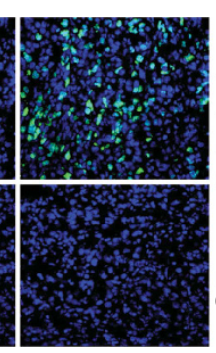

Merge

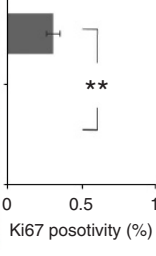

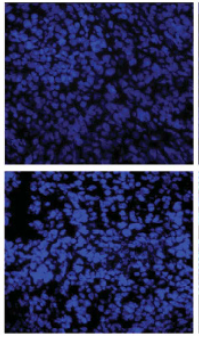

DAPI

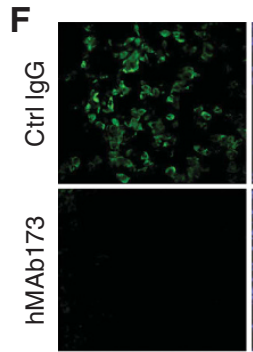

pS6

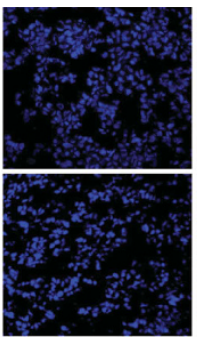

DAPI

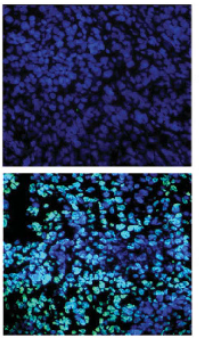

Merge
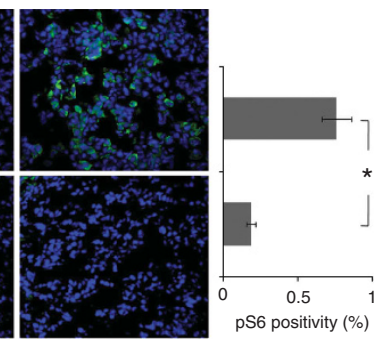

Merge

Figure 5. Evaluation of hMAb173 efficacy in tumour xenograft models. (A and B) Established (A) 786-O and (B) HT29 tumours ( $n=8$ ) were treated with control lgG or hMAb173 at $20 \mathrm{mg} \mathrm{kg}^{-1}, 2$ times a week for 4 weeks. Primary tumour volume was measured 3 times a week. Data are presented as mean \pm s.e.m. P-value was calculated with two-tailed, unpaired Student's t-test using the data from the last time point measurement. (C, E, and F) Immunostaining of harvested primary 786-O tumour with antibodies against (C) Axl, (E) Ki67, and (F) pS6 (Ser235/236). (D) Apoptosis in harvested tumours was analysed with TUNEL assay. Nuclei were counterstained with DAPI. Three tumours from each group were stained and at least three pictures from each sample were taken. Signals were quantified with ImageJ and $P$-value was calculated with two-tailed, unpaired Student's t-test. ${ }^{\star} P<0.02 ;{ }^{* \star} P<0.002$.

Many therapeutics targeting Axl are under development. Recently, three small kinase inhibitors for Axl have entered clinical trials (Sheridan, 2013). However, not all TKIs have activity limited to Axl. The TAM kinases are known to be expressed by macrophages and dendritic cells and inhibit inflammation (Lemke, 2013). In a recent study, deletion of both Axl and Mer led to development of colon cancers induced by inflammatory agents, but loss of only one of these kinases had no effect (Bosurgia et al, 2013). Therefore, a highly specific inhibitor is warranted to avoid severe adverse effects. An alternative approach is to target Axl using highly specific monoclonal antibody. Axl monoclonal antibodies have been developed and reported in a few preclinical studies (Ye et al, 2010; Leconet et al, 2013). We have developed an antibody MAb173 that effectively degrades Axl and shows activity in Axl positive tumour cells to inhibit migration/invasion consistent with the response to siRNA in the same cell lines (Liu et al, 2010). We have humanised MAb173 and confirmed it has low/no immunogenicity to human using $\mathrm{T}$-cell proliferation assay. This antibody induces renal cancer cell apoptosis ex vivo and specifically inhibits Axl-expressing tumour growth in tumour xenograft models in vivo. Tissue analysis shows reduced proliferation, increased cell apoptosis, and inhibited PI3K signalling in tumours treated with hMAb173. Thus, the antibody has direct toxicity to the tumour cells.
This antibody may have even greater efficacy in the immunecompetent host in which antibody can exert antibody-dependent cell-mediated cytotoxicity (ADCC) and induce complement activation. The effect of ADCC has to be measured cautiously, considering Axl is also expressed in tumour stroma. The Axl antibody hMAb173 may cause cytotoxicity to tumour endothelial cells and secondarily cause tumour regression. It may also be toxic to tumour-infiltrating immune cells and modulate tumour growth. We will investigate this question using a surrogate antibody that recognises mouse Axl in a syngeneic mouse tumour model.

\section{ACKNOWLEDGEMENTS}

This work was in part supported by Ezralow Family Foundation and Raymond Mirra Jr Family, and also Nation Natural Science Foundation of China (No: 812721993).

\section{CONFLICT OF INTEREST}

Valery Krasnoperov is employed by VasGene Therapeutics Inc. The authors declare no conflict of interest. 


\section{REFERENCES}

Ben-Batalla I, Schultze A, Wroblewski M, Erdmann R, Heuser M, Waizenegger JS, Riecken K, Binder M, Schewe D, Sawall S, Witzke V, Cubas-Cordova M, Janning M, Wellbrock J, Fehse B, Hagel C, Krauter J, Ganser A, Lorens JB, Fiedler W, Carmeliet P, Pantel K, Bokemeyer C, Loges S (2013) Axl, a prognostic and therapeutic target in acute myeloid leukemia mediates paracrine crosstalk of leukemia cells with bone marrow stroma. Blood 122(14): 2443-2452.

Bosurgi L, Bernink JH, Delgado Cuevas V, Gagliani N, Joannas L, Schmid ET, Booth CJ, Ghosh S, Rothlin CV (2013) Paradoxical role of the protooncogene Axl and Mer receptor tyrosine kinases in colon cancer. Proc Natl Acad Sci USA 110(32): 13091-13096.

Chung BI, Malkowicz SB, Nguyen TB, Libertino JA, McGarvey TW (2003) Expression of the proto-oncongene Axl in renal carcinoma. DNA Cell Biol 22(8): 553-40.

Collett GD, Sage AP, Kirton JP, Alexander MY, Gilmore AP, Canfield AE (2007) Axl/phosphatidylinositol3-kinase signaling inhibits mineral deposition by vascular smooth muscle cells. Circ Res 100(4): 502-509.

Deschavanne PJ, Fertil B (1996) A review of human cell radiosensitivity in vitro. Int J Radiat Oncol Biol Phys 34: 251-266.

Escudier B, Eisen T, Stadler WM, Szczylik C, Oudard S, Siebels M, Negrier S, Chevreau C, Solska E, Desai AA, Rolland F, Demkow T, Hutson TE, Gore M, Freeman S, Schwartz B, Shan M, Simantov R, Bukowski RM. TARGET Study Group (2007a) Sorafenib in advanced clear-cell renal-cell carcinoma. N Engl Med 356(2): 125-134.

Escudier B, Pluzanska A, Koralewski P, Ravaud A, Bracarda S, Szczylik C, Chevreau C, Filipek M, Melichar B, Bajetta E, Gorbunova V, Bay JO, Bodrogi I, Jagiello-Gruszfeld A, Moore N (2007b) Bevacizumab plus interferon alfa-2a for treatment of metastatic renal cell carcinoma: a randomised, double-blind phase III trial. Lancet 370: 2103-2111.

Fridell YW, Jin Y, Quilliam LA, Burchert A, McCloskey P, Spizz G, Varnum B, Der C, Liu ET (1996) Differential activation of the Ras/extracellularsignal-regulated protein kinase pathway is responsible for the biological consequences induced by the Axl receptor tyrosine kinase. Mol Cell Biol 116(1): 135-145.

Gjerdrum C, Tiron C, Høiby T, Stefansson I, Haugen H, Sandal T, Collett K, Li S, McCormack E, Gjertsen BT, Micklem DR, Akslen LA, Glackin C, Lorens JB (2010) Axl is an essential epithelial-to-mesenchymal transitioninduced regulator of breast cancer metastasis and patient survival. Proc Natl Acad Sci USA 107(3): 1124-1129.

Goruppi S, Ruaro E, Varnum B, Schneider C (1999) Gas6-mediated survival in NIH3T3 cells activates stress signaling cascade and is independent of Ras. Oncogene 18(29): 4224-4236.

Gustafsson A, Martuszewska D, Johansson M, Ekman C, Hafizi S, Ljungberg B, Dahlbäck B (2009a) Differential expression of Axl and Gas6 in renal cell carcinoma reflecting tumor advancement and survival. Clin Cancer Res 15(14): 4742-4749.

Gustafsson A, Boström AK, Ljungberg B, Axelson H, Dahlbäck B (2009b) Gas6 and the receptor tyrosine kinase Axl in clear cell renal cell carcinoma. PLoS One 4(10): e7575.

Hafizi S, Alindri F, Karlsson R, Dahlbäck B (2002) Interaction of Axl receptor tyrosine kinase with C1-TEN, a novel C1 domain-containing protein with homology to tensin. Biochem Biophys Res Commun 299(5): 793-800.

Hafizi S, Dahlbäck B (2006) Gas6 and protein S. Vitamin K-dependent ligands for the Axl receptor tyrosine kinase subfamily. FEBS J 273(23): 5231-5244

Han J, Tian R, Yong B, Luo C, Tan P, Shen J, Peng T (2013) Gas6/Axl mediates tumor cell apoptosis, migration and invasion and predicts the clinical outcome of osteosarcoma patients. Biochem Biophys Res Commun 435(3): 493-500.

Harada K, Miyake H, Kumano M, Fujisawa M (2013) Acquired resistance to temsirolimus in human renal cell carcinoma cells is mediated by the constitutive activation of signal transduction pathways through mTORC2. Br J Cancer 109(9): 2389-2395.

Hudes G, Carducci M, Tomczak P, Dutcher J, Figlin R, Kapoor A, Staroslawska E, Sosman J, McDermott D, Bodrogi I, Kovacevic Z, LesovoyV, Schmidt-Wolf IG, Barbarash O, Gokmen E, O'Toole T, Lustgarten S, Moore L, Motzer RJ. Global ARCC Trial (2007) Temsirolimus, interferon alfa, or both for advanced renal-cell carcinoma. N Engl J Med 356: 2271-2281.
Janssen JW, Schulz AS, Steenvoorden AC, Schmidberger M, Strehl S, Ambros PF, Bartram CR (1991) A novel putative tyrosine kinase receptor with oncogenic potential. Oncogene 6: 2113-2120.

Kanesvaran R, Tan MH (2014) Targeted therapy for renal cell carcinoma: the next lap. J Carcinog 13: 3.

Konishi A, Aizawa T, Mohan A, Korshunov VA, Berk BC (2004) Hydrogen peroxide activates the Gas6-Axl pathway in vascular smooth muscle cells. J Biol Chem 279(27): 28766-28770.

Ku BM, Kim SJ, Kim N, Hong D, Choi YB, Lee SH, Gong YD, Kim SY (2014) Transglutaminase 2 inhibitor abrogates renal cell carcinoma in xenograft models. J Cancer Res Clin Oncol 140(5): 757-767.

Lai C, Gore M, Lemke G (1994) Structure, expression, and activity of Tyro 3, a neural adhesion-related receptor tyrosine kinase. Oncogene 9: 2567-2578.

Leconet W, Larbouret C, Chardès T, Thomas G, Neiveyans M, Busson M, Jarlier M, Radosevic-Robin N, Pugnière M, Bernex F, Penault-Llorca F, Pasquet JM, Pèlegrin A, Robert B (2013) Preclinical validation of AXL receptor as a target for antibody-based pancreatic cancer immunotherapy. Oncogene 33(47): 5405-5414.

Lemke G (2013) Biology of the TAM receptors. Cold Spring Harb Perspect Biol 5(11): a009076.

Liu R, Gong M, Li X, Zhou Y, Gao W, Tulpule A, Chaudhary PM, Jung J, Gill PS (2010) Induction, regulation, and biologic function of Axl receptor tyrosine kinase in Kaposi sarcoma. Blood 116(2): 297-305.

Livak KJ, Schmittgen TD (2001) Analysis of relative gene expression data using real-time quantitative PCR and the 2 (-delta deltaC(T)) method. Methods 25(4): 402-408.

Melaragno MG, Fridell YW, Berk BC (1999) The Gas6/Axl system: a novel regulator of vascular cell function. Trends Cardiovasc Med 9(8): 250-253.

Meric F, Lee WP, Sahin A, Zhang H, Kung HJ, Hung MC (2002) Expression profile of tyrosine kinases in breast cancer. Clin Cancer Res 8: 361-367.

Motzer RJ, Escudier B, Oudard S, Hutson TE, Porta C, Bracarda S, Grünwald V, Thompson JA, Figlin RA, Hollaender N, Kay A, Ravaud A. RECORD-1 Study Group (2010) Phase 3 trial of everolimus for metastatic renal cell carcinoma: final results and analysis of prognostic factors. Cancer 116: 4256-4265.

Motzer RJ, Escudier B, Tomczak P, Hutson TE, Michaelson MD, Negrier S, Oudard S, Gore ME, Tarazi J, Hariharan S, Chen C, Rosbrook B, Kim S, Rini BI (2013) Axitinib versus sorafenib as second-line treatment for advanced renal cell carcinoma: overall survival analysis and updated results from a randomized phase 3 trial. Lancet Oncol 14: 552-562.

Motzer RJ, Hutson TE, Tomczak P, Michaelson MD, Bukowski RM, Rixe O, Oudard S, Negrier S, Szczylik C, Kim ST, Chen I, Bycott PW, Baum CM, Figlin RA (2007) Sunitinib versus interferon alfa in metastatic renal-cell carcinoma. N Engl J Med 356: 115-124.

O'Bryan JP, Frye RA, Cogswell PC, Neubauer A, Kitch B, Prokop C, Espinosa 3rd R, Le Beau MM, Earp HS, Liu ET (1991) axl, a transforming gene isolated from primary human myeloid leukemia cells, encodes a novel receptor tyrosine kinase. Mol Cell Biol 11(10): 5016-5031.

Ou WB, Corson JM, Flynn DL, Lu WP, Wise SC, Bueno R, Sugarbaker DJ, Fletcher JA (2011) AXL regulates mesothelioma proliferation and invasiveness. Oncogene 30(14): 1643-1652.

Paccez JD, Vasques GJ, Correa RG, Vasconcellos JF, Duncan K, Gu X, Bhasin M, Libermann TA, Zerbini LF (2013) The receptor tyrosine kinase Axl is an essential regulator of prostate cancer proliferation and tumor growth and represents a new therapeutic target. Oncogene 32(6): 689-698.

Rothlin CV, Ghosh S, Zuniga EI, Oldstone MB, Lemke G (2007) TAM receptors are pleiotropic inhibitors of the innate immune response. Cell 131(6): 1124-1136.

Sheridan C (2013) First Axl inhibitor enters clinical trials. Nat Biotechnol 31(9): 775-776.

Shieh YS, Lai CY, Kao YR, Shiah SG, Chu YW, Lee HS, Wu CW (2005) Expression of axl in lung adenocarcinoma and correlation with tumor progression. Neoplasia 7(12): 1058-1064.

Siegel R, Naishadham D, Jemal A (2013) Cancer statistics, 2013. CA Cancer J Clin 63(1): 11-30.

Sternberg CN, Hawkins RE, Wagstaff J, Salman P, Mardiak J, Barrios CH, Zarba JJ, Gladkov OA, Lee E, Szczylik C, McCann L, Rubin SD, Chen M, Davis ID (2013) A randomized, double-blind phase III study of pazopanib in patients with advanced and/or metastatic renal cell carcinoma: final overall survival results and safety update. Eur J Cancer 49: 1287-1296.

Wu CW, Li AF, Chi CW, Lai CH, Huang CL, Lo SS, Lui WY, Lin WC (2002) Clinical significance of AXL kinase family in gastric cancer. Anticancer Res 22(2B): 1071-1078. 
Yanagita M, Arai H, Nakano T, Ohashi K, Mizuno K, Fukatsu A, Doi T, Kita T (2001) Gas6 induces mesangial cell proliferation via latent transcription factor STAT3. J Biol Chem 276(45): 42364-42369.

Ye X, Li Y, Stawicki S, Couto S, Eastham-Anderson J, Kallop D, Weimer R, Wu Y, Pei L (2010) An anti-Axl monoclonal antibody attenuates xenograft tumor growth and enhances the effect of multiple anticancer therapies. Oncogene 29(38): 5254-5264.

Zhang Y, Li N, Chen Q, Yan K, Liu Z, Zhang X, Liu P, Chen Y, Han D (2013) Breakdown of immune homeostasis in the testis of mice lacking Tyro3, Axl and Mer receptor tyrosine kinases. Immunol Cell Biol 91(6): 416-426.

Zhang YX, Knyazev PG, Cheburkin YV, Sharma K, Knyazev YP, Orfi L, Szabadkai I, Daub H, Kéri G, Ullrich A (2008) AXL is a potential target for therapeutic intervention in breast cancer progression. Cancer Res 68(6): 1905-1915.
Zhang Z, Lee JC, Lin L, Olivas V, Au V, LaFramboise T, Abdel-Rahman M, Wang X, Levine AD, Rho JK, Choi YJ, Choi CM, Kim SW, Jang SJ, Park YS, Kim WS, Lee DH, Lee JS, Miller VA, Arcila M, Ladanyi M, Moonsamy P, Sawyers C, Boggon TJ, Ma PC, Costa C, Taron M, Rosell R, Halmos B, Bivona TG (2012) Activation of the AXL kinase causes resistance to EGFR-targeted therapy in lung cancer. Nat Genet 44(8): 852-860.

This work is published under the standard license to publish agreement. After 12 months the work will become freely available and the license terms will switch to a Creative Commons AttributionNonCommercial-Share Alike 4.0 Unported License.

Supplementary Information accompanies this paper on British Journal of Cancer website (http://www.nature.com/bjc) 